\title{
Avaliação do Seguimento de um Ano dos Pacientes Incluídos no Registro Brasileiro de Síndromes Coronárias Agudas (ACCEPT)
}

One year follow-up Assessment of Patients Included in the Brazilian Registry of Acute Coronary Syndromes (ACCEPT)

Pedro Gabriel Melo de Barros e Silva, ${ }^{1,2}$ Otavio Berwanger, ${ }^{3}$ Elizabete Silva dos Santos, ${ }^{4}$ Antônio Carlos Sobral Sousa, ${ }^{5,6}$ Margaret Assad Cavalcante, ${ }^{7,8}$ Pedro Beraldo de Andrade, ${ }^{9.10}$ Fernando Carvalho Neuenschwander, ${ }^{11}$ Hugo Vargas Filho, ${ }^{12}$ Jorge Ilha Guimarães, ${ }^{13}$ Jadelson Andrade, ${ }^{14}$ Angelo Amato Vincenzo de Paola, ${ }^{15}$ Marcus Vinicius Bolivar Malachias, ${ }^{16,17}$ Luiz Alberto Piva e Mattos, ${ }^{13,18,19}$ Dalton Bertolim Precoma, ${ }^{20,21}$ Fernando Bacal, ${ }^{22}$ Oscar Pereira Dutra ${ }^{23}$ Hospital do Coração - Pesquisa Clínica, ${ }^{1}$ São Paulo, SP - Brasil Hospital Samaritano Paulista - Cardiologia, ${ }^{2}$ São Paulo, SP - Brasil

Hospital Israelita Albert Einstein, ${ }^{3}$ São Paulo, SP - Brasil

Instituto Dante Pazzanese de Cardiologia, ${ }^{4}$ São Paulo, SP - Brasil

PPGCS e HU da Universidade Federal de Sergipe (UFS), ${ }^{5}$ Aracaju, SE - Brasil

Hospital São Lucas - Rede D 'Or, ${ }^{6}$ Aracaju, SE - Brasil

Universidade do Oeste Paulista (Unoeste), ${ }^{7}$ Presidente Prudente, SP - Brasil

Hospital Regional de Presidente Prudente, ${ }^{8}$ Presidente Prudente, SP - Brasil

Faculdade de Medicina de Marilia, ${ }^{9}$ Marilia, SP - Brasil

Santa Casa de Marília, ${ }^{10}$ Marília, SP - Brasil

Hospital Vera Cruz, ${ }^{11}$ Belo Horizonte, MG - Brasil

Hospital São Vicente de Paulo, ${ }^{12}$ Passo Fundo, RS - Brasil

Sociedade Brasileira de Cardiologia, ${ }^{13}$ Rio de Janeiro, RJ - Brasil

Hospital da Bahia, ${ }^{14}$ Salvador, BA - Brasil

Universidade Federal de São Paulo Escola Paulista de Medicina, ${ }^{15}$ São Paulo, SP - Brasil

Faculdade de Ciências Médicas de Minas Gerais, ${ }^{16}$ Belo Horizonte, MG - Brasil

Instituto de Hipertensão Arterial - Diretoria Clínica, ${ }^{17}$ Belo Horizonte, MG - Brasil

Rede D 'Or, São Paulo, ${ }^{18}$ SP - Brasil

Rede D 'Or, Recife, ${ }^{19}$ PE - Brasil

Pontifícia Universidade Católica do Paraná - Escola de Medicina, ${ }^{20}$ Curitiba, PR - Brasil

Sociedade Hospitalar Angelina Caron - Cardiologia, 21 Campina Grande do Sul, PR - Brasil

Universidade de São Paulo Faculdade de Medicina Hospital das Clínicas Instituto do Coração, 22 São Paulo, SP - Brasil

Instituto de Cardiologia - Fundação Universitária de Cardiologia do Rio Grande do Sul, ${ }^{23}$ Porto Alegre, RS - Brasil

\section{Resumo}

Fundamento: Existe carência de informações prospectivas sobre a evolução em um ano após uma síndrome coronária aguda (SCA) em uma grande amostra de pacientes brasileiros.

Objetivos: Avaliar a prescrição de terapias baseadas em evidência, a ocorrência de desfechos graves e os preditores para estes desfechos em um registro brasileiro multicêntrico de pacientes com SCA.

Métodos: O ACCEPT é um estudo observacional prospectivo que incluiu pacientes internados com diagnóstico de SCA em 47 hospitais brasileiros. Os pacientes foram seguidos por 1 ano e coletou-se dados sobre prescrição médica e ocorrência de eventos cardiovasculares maiores (mortalidade cardiovascular, reinfarto e acidente vascular encefálico - AVE). Valores de p < 0,05 foram considerados estatisticamente significantes.

Resultados: Um total de 5.047 pacientes foram incluídos neste registro, de agosto de 2010 até abril de 2014. Foi confirmado o diagnóstico de SCA em 4.782 pacientes $(94,7 \%)$ e, dentre os 3 diagnósticos possíveis, o mais comum foi SCA com elevação do segmento ST (35,8\%). A taxa de eventos cardiovasculares maiores foi de 13,6 \% em 1 ano. A prescrição completa de terapias baseadas em evidência na admissão hospitalar foi de 62,1\%. Idade, atendimento público, infarto agudo do miocárdio, AVE, insuficiência renal, diabetes e qualidade da terapia estiveram associados de forma independente à ocorrência de eventos cardiovasculares maiores.

Correspondência: Pedro Gabriel Melo de Barros e Silva •

Hospital Samaritano Paulista - R. Dr. Fausto Ferraz, 204-232. CEP 01333-030, Bela Vista, SP - Brasil

E-mail: pgabriel@prestadores.samaritanopaulista.com.br

Artigo recebido em 10/12/2019, revisado em 09/03/2020, aceito em 08/04/2020

DOI: https://doi.org/10.36660/abc.20190879 
Conclusões: No seguimento de 1 ano do registro ACCEPT, mais de $10 \%$ dos pacientes apresentaram eventos cardiovasculares maiores e esta taxa variou de acordo com a qualidade da terapia. Há necessidade da elaboração de estratégias para melhorar o uso de terapias baseadas em evidência no sentido de minimizar os eventos cardiovasculares na população brasileira. (Arq Bras Cardiol. 2020; 114(6):995-1003)

Palavras-chave: Síndrome Coronariana Aguda; Infarto do Miocárdio; Fatores de Risco; Estudos Multicêntricos; Registros Médicos/estatística \&dados numéricos.

\begin{abstract}
Background: There is lack of prospective data on evolution within one year of acute coronary syndromes (ACS) in a representative population of Brazilian patients.
\end{abstract}

Objectives: To assess the prescription of evidence-based therapies, the incidence of severe outcomes and the predictors for these outcomes in a multicenter Brazilian registry of ACS patients.

Methods: The ACCEPT is a prospective observational study, which included patients hospitalized with a diagnostic of ACS in 47 Brazilian hospitals. The patients were followed for a 1 year and data were collected on the medical prescription and the occurrence of major cardiovascular events (cardiovascular mortality, reinfarction and cerebrovascular accident - CVA). Values of $p<0.05$ were considered statistically significant.

Results: A total of 5,047 patients were included in this registry from August 2010 to April 2014. The diagnosis of ACS was confirmed in 4,782 patients (94.7\%) and, among those, the most frequent diagnosis was ACS with ST segment elevation (35.8\%). The rate of major cardiovascular events was $13.6 \%$ within 1 year. Adherence to prescription of evidence-based therapy at admission was of $62.1 \%$. Age, public service, acute myocardial infarction, CVA, renal failure, diabetes and quality of therapy were associated independently with the occurrence of major cardiovascular events.

Conclusions: During the one-year follow-up of the ACCEPT registry, more than $10 \%$ of the patients had major cardiovascular events and this rate ranged according with the quality of therapy. Strategies must be elaborated to improve the use of evidence-based therapies to minimize the cardiovascular events among the Brazilian population. (Arq Bras Cardiol. 2020; 114(6):995-1003)

Keywords: Acute Coronary Syndrome; Myocardial Infarction; Risk factors; Medical Records/ statistics\& numeral data; Multicenter Studies

Full texts in English - http://www.arquivosonline.com.br

\section{Introdução}

O grupo das doenças cardiovasculares, particularmente a síndrome coronária aguda (SCA), representa a principal causa de mortalidade e incapacidade no Brasil e no mundo. ${ }^{1-3}$ Além da elevada frequência atual, há perspectiva de crescimento deste grupo de doenças em países em desenvolvimento como o Brasil. ${ }^{1-5}$ Apesar da elevada morbimortalidade da SCA no cenário atual, diversas estratégias de comprovada eficácia para reduzir o risco de complicações nestes pacientes têm sido desenvolvidas. ${ }^{6,7}$ Entretanto, há falhas na aplicação das terapias baseadas em evidência em pacientes com SCA, conforme identificado em registros prévios de prática clínica. ${ }^{8-10}$ Tais registros multicêntricos avaliaram predominantemente o período intra-hospitalar ou decorridos 30 dias do evento agudo registrando-se, todavia, a carência de informações a longo prazo sobre o acompanhamento destes pacientes. ${ }^{8-10}$ Dentre estes registros prévios com seguimento de até 30 dias, há a publicação de dados parciais (sem amostra completa) do estudo ACCEPT. ${ }^{10}$ Conforme previamente reportado na publicação dos dados parciais de 30 dias. $^{10}$ o ACCEPT tinha como plano continuar o estudo, arrolando um número maior de pacientes e incluir dados do seguimento de 12 meses. Dessa forma, a presente análise realizou, novamente, a avaliação das características basais e a adesão inicial da prescrição médica a terapias baseadas em evidências numa população maior (aproximadamente o dobro de pacientes em relação à publicação inicial com dados intermediários) e incluiu dados sobre a taxa de ocorrência de desfechos clínicos graves durante o seguimento.

\section{Objetivos}

Além dos resultados finais em 30 dias com a população completa do estudo, esta análise do seguimento de 1 ano tem os seguintes objetivos específicos:

- Avaliar a taxa de eventos cardiovasculares maiores em 12 meses numa população de pacientes brasileiros pós-SCA;

- Avaliar a taxa de conformidade da prescrição médica a terapias baseadas em evidência em 12 meses numa população de pacientes brasileiros pós-SCA;

- Identificar preditores de eventos cardiovasculares maiores em 12 meses numa população de pacientes brasileiros pós-SCA.

\section{Métodos}

\section{Delineamento do estudo}

O Registro ACCEPT (Acute Coronary Care Evaluation of Practice Registry) é um projeto idealizado pela Sociedade Brasileira de Cardiologia (SBC), cujos métodos foram previamente publicados. ${ }^{10,11}$ De forma resumida, trata-se de pesquisa prospectiva, voluntária, multicêntrica que reuniu 53 centros das 5 regiões brasileiras com a seguinte distribuição: sudeste $(50,9 \%)$, nordeste $(13,2 \%)$, sul $(24,5 \%)$, centro oeste $(5,7 \%)$ e norte $(5,7 \%)$. A inclusão de pacientes ocorreu de agosto de 2010 até abril de 2014, em centros hospitalares com assistência pública (Sistema Único de Saúde - SUS), de saúde suplementar (operadoras de saúde) ou privados, de acordo com a seguinte distribuição: SUS 2669/4782 (55,8\%), saúde suplementar 1968/4782 (41,2\%) e privado 145/4782 (3\%). 


\section{Participantes do estudo}

Foram incluídos pacientes na vigência do diagnóstico de SCA nas suas diferentes formas de apresentação: angina instável (AI), infarto agudo do miocárdio sem supradesnível do segmento ST (IAM sSST) e com supradesnível do segmento ST (IAM cSST). Os principais critérios de inclusão foram sintomas isquêmicos suspeitos de SCA associados a alterações no eletrocardiograma (ECG) compatíveis com alteração isquêmica e/ou marcadores de lesão miocárdica, acima do limite superior da normalidade. Foram excluídos pacientes transferidos de outras instituições com mais de 12 horas do início dos sintomas.

\section{Procedimentos do estudo e variáveis analisadas}

Os procedimentos do estudo e variáveis analisadas no estudo ACCEPT foram previamente publicados. ${ }^{10,11}$ De forma sucinta, a coleta de dados ocorreu na internação, com dados da admissão (visita índice), e também foi feita uma segunda coleta de dados de 7 dias ou até a alta hospitalar (o que ocorresse primeiro). Após estas duas primeiras visitas, o estudo planejou visitas de 30 dias, 6 meses e 12 meses, que poderiam ocorrer de forma presencial na rotina assistencial ou por telefone.

Tendo a característica de um estudo pragmático, a identificação de comorbidade dos pacientes (ex.: hipertensão arterial, dislipidemia) poderia ser realizada da seguinte forma: relato pelo paciente, uso de medicamento (anti-hipertensivo, hipolipemiante) ou avaliação do investigador (neste último, os centros foram orientados a seguirem as recomendações de critérios diagnósticos adotadas pelas diretrizes vigentes da Sociedade Brasileira de Cardiologia). Características do exame físico poderiam ser obtidas por mensuração direta (a obesidade foi definida por IMC $>30 \mathrm{Kg} / \mathrm{m}^{2}$ ). Demais critérios se basearam no registro em prontuário de uma variável coletada através de questionamento em entrevista (ex.: estresse, ex-tabagista se cessação $>6$ meses).

$\mathrm{O}$ esquema terapêutico baseado em evidência que foi considerado no ACCEPT não se modificou durante o estudo e se baseou em diretrizes vigentes..$^{6,7}$ Este esquema terapêutico pode ser dividido da seguinte forma:

- Internação do evento índice: Dupla antiagregação, anticoagulante parenteral, estatina e betabloqueador com adição da terapia de reperfusão nos casos de IAM cSST.

- Terapia ambulatorial (pós-alta): Dupla antiagregação, estatina, betabloqueador e IECA/BRA.

Os desfechos cardiovasculares de interesse analisados na população incluída foram: mortalidade cardiovascular, parada cardíaca não fatal, reinfarto e acidente vascular encefálico (AVE). ${ }^{10,11}$ Estes desfechos foram reportados pelo investigador de acordo com critérios recomendados, ${ }^{10,11} \mathrm{sem}$ utilização de um comitê independente para adjudicação de eventos.

\section{Análise estatística}

A avaliação da normalidade de distribuição de variáveis contínuas foi realizada através de histogramas. Variáveis contínuas de distribuição normal foram descritas como média \pm desvio padrão. As médias foram comparadas entre os três grupos de diagnósticos utilizando a análise de variância (Anova). Variáveis categóricas foram descritas como frequências absolutas e relativas. Proporções foram comparadas pelo teste de Qui-quadrado ou o Teste (exato) de Fisher-Freeman-Halton. Modelos de Equações de Estimação Generalizada (EEG) foram utilizados para avaliar a terapia medicamentosa ao longo do tempo. Com a finalidade de comparar os eventos cardiovasculares maiores, de acordo com o diagnóstico final, foram utilizados o modelo de riscos proporcionais de Cox e o gráfico de Kaplan-Meier. A identificação de preditores independentes de eventos combinados (AVE, reinfarto e óbito) foi realizada por modelos de risco proporcionais de Cox com diagnóstico final e o fator baseline avaliado. Esta análise de preditores foi realizada inicialmente de forma univariada e as variáveis com valor de $\mathrm{p}<0,15$ foram incluídas na análise multivariada. Valores de $p$ apresentados são do tipo bilateral e $p<0,05$ foi considerado estatisticamente significante nas análises finais. Adicionalmente, foi realizado teste de interação entre as variáveis selecionadas na análise multivariada. Todas as análises foram realizadas com auxílio do programa $\mathrm{R}$, versão 3.6.1.

\section{Resultados}

Entre agosto de 2010 e abril de 2014, 5.047 pacientes foram recrutados nesse registro nacional, sendo 265 destes $(5,25 \%)$ portadores de dor torácica a esclarecer e excluídos do seguimento clínico por não preencherem os critérios de inclusão da pesquisa. Sendo assim, 4.782 pacientes portadores de SCA foram efetivamente incluídos na análise e seguidos neste registro prospectivo, em 53 centros hospitalares das 5 regiões brasileiras. Em um total de 410 pacientes $(8,6 \%)$ não foi possível obter informação final de 12 meses.

\section{Características basais}

O perfil clínico dos pacientes evidenciou a inclusão de aproximadamente $70 \%$ dos pacientes com diagnóstico de IAM na admissão, quase um terço de diabéticos, e próximo de $90 \%$ com a evidência de ao menos um fator de risco presente, sendo hipertensão arterial sistêmica o mais comum deles (Tabela 1).

\section{Adesão da prescrição médica a terapias baseadas em evidência}

A prescrição adotada logo após a admissão demonstra que a adesão completa aos medicamentos recomendados nas diretrizes vigentes foi de 62,1\% (tabela 2). Esta adesão inclui dupla terapia antiplaquetária (aspirina/inibidor da P2Y12) associada a anticoagulante parenteral, estatina e betabloqueador.

Dos 1.714 pacientes que apresentaram IAMcSST, alguma modalidade de reperfusão do miocárdio (fibrinólise ou intervenção coronariana percutânea primária) foi realizada em $1.412(82,4 \%)$ indivíduos. Em análise da prescrição das terapias de reperfusão no IAM, observamse percentuais distintos e decrescentes, de acordo com a 
Tabela 1 - Características basais dos pacientes incluídos de acordo com tipo de síndrome coronária aguda

\begin{tabular}{|c|c|c|c|c|c|}
\hline \multicolumn{6}{|c|}{ Diagnóstico Final do Paciente } \\
\hline & $\begin{array}{l}\text { Angina Instável } \\
\qquad(\mathrm{n}=1453)\end{array}$ & $\begin{array}{l}\text { IAM sem supra de } \\
\text { ST }(n=1615)\end{array}$ & $\begin{array}{l}\text { IAM com supra de } \\
\text { ST }(n=1714)\end{array}$ & Total $(n=4782)$ & Valor de $p$ \\
\hline Idade; média $\pm \mathrm{DP}$ & $63,9 \pm 11,9(n=1449)$ & $64,7 \pm 12,4(n=1603)$ & $60,8 \pm 12,4(n=1702)$ & $63,1 \pm 12,4(n=4754)$ & $<0,001^{(1)}$ \\
\hline Sexo (Feminino) & $588 / 1453(40,5 \%)$ & $489 / 1615(30,3 \%)$ & $460 / 1714(26,8 \%)$ & $1537 / 4782(32,1 \%)$ & $<0,001$ \\
\hline Transferido de outro serviço (Sim) & $179 / 1451(12,3 \%)$ & $393 / 1614(24,3 \%)$ & $803 / 1713(46,9 \%)$ & $1375 / 4778(28,8 \%)$ & $<0,001$ \\
\hline Atendimento (Saúde Suplementar/Particular) & $757 / 1453(52,1 \%)$ & $775 / 1615(48 \%)$ & $581 / 1714(33,9 \%)$ & $2113 / 4782(44,2 \%)$ & $<0,001$ \\
\hline Pressão Arterial Sistólica; média \pm DP & $138,1 \pm 24,1(n=1452)$ & $137,9 \pm 28(n=1615)$ & $131,5 \pm 26(n=1713)$ & $135,7 \pm 26,4(n=4780)$ & $<0,001^{(1)}$ \\
\hline Pressão Arterial Diastólica; média \pm DP & $81,4 \pm 13,9(n=1452)$ & $81,3 \pm 16,4(n=1615)$ & $80,4 \pm 16,4(n=1713)$ & $81 \pm 15,7(n=4780)$ & $0,142^{(1)}$ \\
\hline Frequência Cardíaca ; média \pm DP & $74,6 \pm 15,3(n=1452)$ & $77,6 \pm 18(n=1615)$ & $79,4 \pm 17,2(n=1713)$ & $77,4 \pm 17(n=4780)$ & $<0,001^{(1)}$ \\
\hline Dislipidemia & $971 / 1453(66,8 \%)$ & $915 / 1615(56,7 \%)$ & $734 / 1713(42,8 \%)$ & $2620 / 4781(54,8 \%)$ & $<0,001$ \\
\hline IAM prévio & $507 / 1451(34,9 \%)$ & $535 / 1614(33,1 \%)$ & $267 / 1713(15,6 \%)$ & $1309 / 4778(27,4 \%)$ & $<0,001$ \\
\hline História de Angina & $774 / 1452(53,3 \%)$ & $554 / 1614(34,3 \%)$ & $406 / 1713(23,7 \%)$ & $1734 / 4779(36,3 \%)$ & $<0,001$ \\
\hline Hipertensão & $1197 / 1453(82,4 \%)$ & $1252 / 1615(77,5 \%)$ & $1116 / 1713(65,1 \%)$ & $3565 / 4781(74,6 \%)$ & $<0,001$ \\
\hline História familiar de doença coronária & $643 / 1453(44,3 \%)$ & $658 / 1615(40,7 \%)$ & $699 / 1713(40,8 \%)$ & $2000 / 4781(41,8 \%)$ & 0,081 \\
\hline AVE & $137 / 1453(9,4 \%)$ & $125 / 1615(7,7 \%)$ & $98 / 1713(5,7 \%)$ & $360 / 4781(7,5 \%)$ & $<0,001$ \\
\hline Estresse e/ou Depressão & $506 / 1451(34,9 \%)$ & $419 / 1614(26 \%)$ & $466 / 1713(27,2 \%)$ & $1391 / 4778(29,1 \%)$ & $<0,001$ \\
\hline Insuficiência renal & $88 / 1452(6,1 \%)$ & $99 / 1615(6,1 \%)$ & $72 / 1713(4,2 \%)$ & $259 / 4780(5,4 \%)$ & 0,021 \\
\hline Diabetes Mellitus & $477 / 1453(32,8 \%)$ & $582 / 1615(36 \%)$ & $453 / 1713(26,4 \%)$ & $1512 / 4781(31,6 \%)$ & $<0,001$ \\
\hline Diabetes em uso de insulina & $134 / 474(28,3 \%)$ & $150 / 582(25,8 \%)$ & $84 / 453(18,5 \%)$ & $368 / 1509(24,4 \%)$ & - \\
\hline Insuficiência cardíaca & $180 / 1452(12,4 \%)$ & $156 / 1615(9,7 \%)$ & $87 / 1713(5,1 \%)$ & $423 / 4780(8,8 \%)$ & $<0,001$ \\
\hline Intervenção coronária percutânea & $489 / 1450(33,7 \%)$ & $406 / 1614(25,2 \%)$ & $209 / 1713(12,2 \%)$ & $1104 / 4777(23,1 \%)$ & $<0,001$ \\
\hline Cirurgia de RM & $223 / 1452(15,4 \%)$ & $213 / 1615(13,2 \%)$ & $68 / 1713(4 \%)$ & $504 / 4780(10,5 \%)$ & $<0,001$ \\
\hline Uso prévio de AAS & $861 / 1453(59,3 \%)$ & $703 / 1615(43,5 \%)$ & $383 / 1713(22,4 \%)$ & $1947 / 4781(40,7 \%)$ & $<0,001$ \\
\hline Obesidade Abdominal & $531 / 1452(36,6 \%)$ & $552 / 1615(34,2 \%)$ & $521 / 1713(30,4 \%)$ & $1604 / 4780(33,6 \%)$ & 0,001 \\
\hline Sedentarismo & $949 / 1453(65,3 \%)$ & $968 / 1615(59,9 \%)$ & $962 / 1713(56,2 \%)$ & $2879 / 4781(60,2 \%)$ & $<0,001$ \\
\hline Doença arterial periférica & $130 / 1453(8,9 \%)$ & $135 / 1615(8,4 \%)$ & $126 / 1713(7,4 \%)$ & $391 / 4781(8,2 \%)$ & 0,252 \\
\hline \multicolumn{6}{|l|}{ Tabagismo } \\
\hline Nunca & $761 / 1453(52,4 \%)$ & $756 / 1615(46,8 \%)$ & $664 / 1713(38,8 \%)$ & $2181 / 4781(45,6 \%)$ & $<0,001$ \\
\hline Ex-tabagista & $487 / 1453(33,5 \%)$ & $503 / 1615(31,1 \%)$ & $387 / 1713(22,6 \%)$ & $1377 / 4781(28,8 \%)$ & \\
\hline Atual & $205 / 1453(14,1 \%)$ & $356 / 1615(22 \%)$ & $662 / 1713(38,6 \%)$ & $1223 / 4781(25,6 \%)$ & \\
\hline
\end{tabular}

Valor de p: Teste Qui-quadrado. (1) Teste ANOVA.

região federativa brasileira: $87,3 \%, 84,5 \%, 72,8 \%, 66,7 \%$ e $65,7 \%$, ( $p<0,001)$, nas regiões sul, sudeste, nordeste, centro-oeste e norte do Brasil, respectivamente. Na medida da elevação da gravidade, na apresentação clínica desses três componentes das SCA, observou-se uma progressiva elevação da prescrição "estratégias invasivas", seja de cinecoronariografia $(68,0 \%, 83,1 \%$ e $90,4 \% ; p<0,001)$, bem como da efetiva realização de procedimento de revascularização do miocárdio (38,2\%, 54,4\% e 76,4\%; p < $0,001)$, nos casos de angina instável, IAM SSST e IAM CSST, respectivamente. O procedimento de revascularização preferencial nesses pacientes foi a intervenção coronária percutânea com taxas superiores a 95\% da utilização de stents coronários em pacientes tratados de forma percutânea. O percentual de revascularização percutânea dentre todos os pacientes com SCA variou conforme o diagnóstico: angina instável, IAM SSST e IAM CSST (33,6\%, $47,4 \%$ e $75,1 \%$, respectivamente; $p<0,001$ ).

$\mathrm{Na}$ alta hospitalar, observou-se que a prescrição de um inibidor da P2Y12 variou conforme o tipo de SCA $(66,4 \%$ 
Tabela 2 - Utilização de medicamentos na fase de admissão dos pacientes com síndrome coronária aguda

\begin{tabular}{|c|c|c|c|c|c|}
\hline Medicação & Angina Instável & $\begin{array}{l}\text { IAM sem supra } \\
\text { de ST }\end{array}$ & $\begin{array}{l}\text { IAM com supra } \\
\text { de ST }\end{array}$ & Total & $p$ \\
\hline AAS & $1399 / 1449(96,5 \%)$ & $1580 / 1615(97,8 \%)$ & $1688 / 1713(98,5 \%)$ & $4667 / 4777(97,7 \%)$ & 0,001 \\
\hline Betabloqueador & $1144 / 1449(79 \%)$ & $1323 / 1615(81,9 \%)$ & $1352 / 1713(78,9 \%)$ & $3819 / 4777(79,9 \%)$ & 0,052 \\
\hline Inibidor P2Y12 & $1239 / 1449(85,5 \%)$ & $1483 / 1615(91,8 \%)$ & $1671 / 1713(97,5 \%)$ & $4393 / 4777(92 \%)$ & $<0,001$ \\
\hline Clopidogrel & $1213 / 1449(83,7 \%)$ & $1401 / 1615(86,7 \%)$ & $1531 / 1713(89,4 \%)$ & $4145 / 4777(86,8 \%)$ & $<0,001$ \\
\hline Prasugrel & $11 / 1449(0,8 \%)$ & $17 / 1615(1,1 \%)$ & $15 / 1713(0,9 \%)$ & $43 / 4777(0,9 \%)$ & 0,685 \\
\hline Ticagrelor & $23 / 1449(1,6 \%)$ & $80 / 1615(5 \%)$ & $149 / 1713(8,7 \%)$ & $252 / 4777(5,3 \%)$ & $<0,001$ \\
\hline Anticoagulante Parenteral & $1151 / 1449(79,4 \%)$ & $1468 / 1615(90,9 \%)$ & $1500 / 1713(87,6 \%)$ & $4119 / 4777(86,2 \%)$ & $<0,001$ \\
\hline Enoxaparina & $837 / 1449(57,8 \%)$ & $1039 / 1615(64,3 \%)$ & $1086 / 1713(63,4 \%)$ & $2962 / 4777(62 \%)$ & $<0,001$ \\
\hline Fondaparinux & $113 / 1449(7,8 \%)$ & $206 / 1615(12,8 \%)$ & $174 / 1713(10,2 \%)$ & $493 / 4777(10,3 \%)$ & $<0,001$ \\
\hline Heparina não-fracionada & $214 / 1449(14,8 \%)$ & $240 / 1615(14,9 \%)$ & $282 / 1713(16,5 \%)$ & $736 / 4777(15,4 \%)$ & 0,319 \\
\hline Inibidores da GPIIb/IIla & $23 / 1449(1,6 \%)$ & $91 / 1615(5,6 \%)$ & $292 / 1713(17 \%)$ & $406 / 4777(8,5 \%)$ & $<0,001$ \\
\hline Abciximab & $3 / 1449(0,2 \%)$ & $10 / 1615(0,6 \%)$ & $119 / 1713(6,9 \%)$ & $132 / 4777(2,8 \%)$ & $<0,001$ \\
\hline Tirofiban & $20 / 1449(1,4 \%)$ & $82 / 1615(5,1 \%)$ & $173 / 1713(10,1 \%)$ & $275 / 4777(5,8 \%)$ & $<0,001$ \\
\hline Inibidor da ECA & $890 / 1449(61,4 \%)$ & $1059 / 1615(65,6 \%)$ & $1263 / 1713(73,7 \%)$ & $3212 / 4777(67,2 \%)$ & $<0,001$ \\
\hline Estatina & $1302 / 1449(89,9 \%)$ & $1467 / 1615(90,8 \%)$ & $1576 / 1713(92 \%)$ & $4345 / 4777(91 \%)$ & 0,108 \\
\hline Lovastatina & $0 / 1293(0 \%)$ & $0 / 1461(0 \%)$ & $1 / 1568(0,1 \%)$ & $1 / 4322(0 \%)$ & \\
\hline Pravastatina & $40 / 1293(3,1 \%)$ & $44 / 1461(3 \%)$ & $56 / 1568(3,6 \%)$ & $140 / 4322(3,2 \%)$ & \\
\hline Sinvastatina & $581 / 1293(44,9 \%)$ & $619 / 1461(42,4 \%)$ & $914 / 1568(58,3 \%)$ & $2114 / 4322(48,9 \%)$ & \\
\hline Rosuvastatina & $102 / 1293(7,9 \%)$ & 103/1461 (7\%) & $60 / 1568(3,8 \%)$ & $265 / 4322(6,1 \%)$ & \\
\hline Atorvastatina & $570 / 1293(44,1 \%)$ & $695 / 1461(47,6 \%)$ & $537 / 1568(34,2 \%)$ & $1802 / 4322(41,7 \%)$ & \\
\hline Dupla Terapia antiplaquetária & $1211 / 1449(83,6 \%)$ & $1463 / 1615(90,6 \%)$ & $1649 / 1713(96,3 \%)$ & $4323 / 4777(90,5 \%)$ & $<0,001$ \\
\hline Terapia completa & $787 / 1449(54,3 \%)$ & $1062 / 1615(65,8 \%)$ & $1116 / 1713(65,1 \%)$ & $2965 / 4777(62,1 \%)$ & $<0,001$ \\
\hline
\end{tabular}

na angina instável, $77,7 \%$ no IAM SSST e 90,9\% no IAM CSST; $\mathrm{p}<0,001)$, e o tipo de tratamento da doença coronária (ICP (94,2\%), cirurgia (25\%) ou clínico (66,2\%); p <0,001).

A evolução das principais terapias da admissão para a alta hospitalar, ao final de 30 dias e em 6 e 12 meses demonstra redução progressiva no uso das terapias recomendadas, especialmente do uso de inibidores do receptor P2Y12 (Figura 1).

\section{Desfechos clínicos}

Os desfechos clínicos foram mensurados cumulativamente ao final dos 12 meses de evolução (Figura 2). Dentre portadores de $\mathrm{Al}$, não foi identificada uma associação entre ocorrência de eventos combinados (mortalidade, reinfarto ou AVE), ao final de 12 meses, e realização de procedimento de revascularização do miocárdio (Tabela 3). Na evidência de IAM SSST, observou-se redução significativa da ocorrência de eventos cardiovasculares maiores, inclusive mortalidade cardiovascular, dentre aqueles submetidos ou não a um procedimento de revascularização do miocárdio, respectivamente (mortalidade $=6,29$ por 100 pessoas ano versus 12,06 por 100 pessoas ano; $p<0,001$ e desfechos cardiovasculares maiores $=13,18$ por 100 pessoas ano versus 17,96 por 100 pacientes-ano; $p=0,038$ ). Pacientes acometidos por IAM CSST apresentaram significativa redução das taxas de mortalidade e de ocorrência de eventos cardiovasculares maiores quando submetidos a revascularização do miocárdio (mortalidade $=8,02$ por 100 pessoas ano versus 18,54 por 100 pessoas ano; $p<0,001$ e eventos cardiovasculares $=13,11$ por 100 pessoas anos versus 21,69 por 100 pessoas anos; $\mathrm{p}<0,001$ ). Em análise multivariada (Tabela 4), os seguintes fatores estiveram associados à ocorrência de eventos cardiovasculares maiores: idade, atendimento público, IAM, AVE, insuficiência renal, diabetes e qualidade da terapia (completa ou não). Não houve interação significativa entre as covariáveis. 


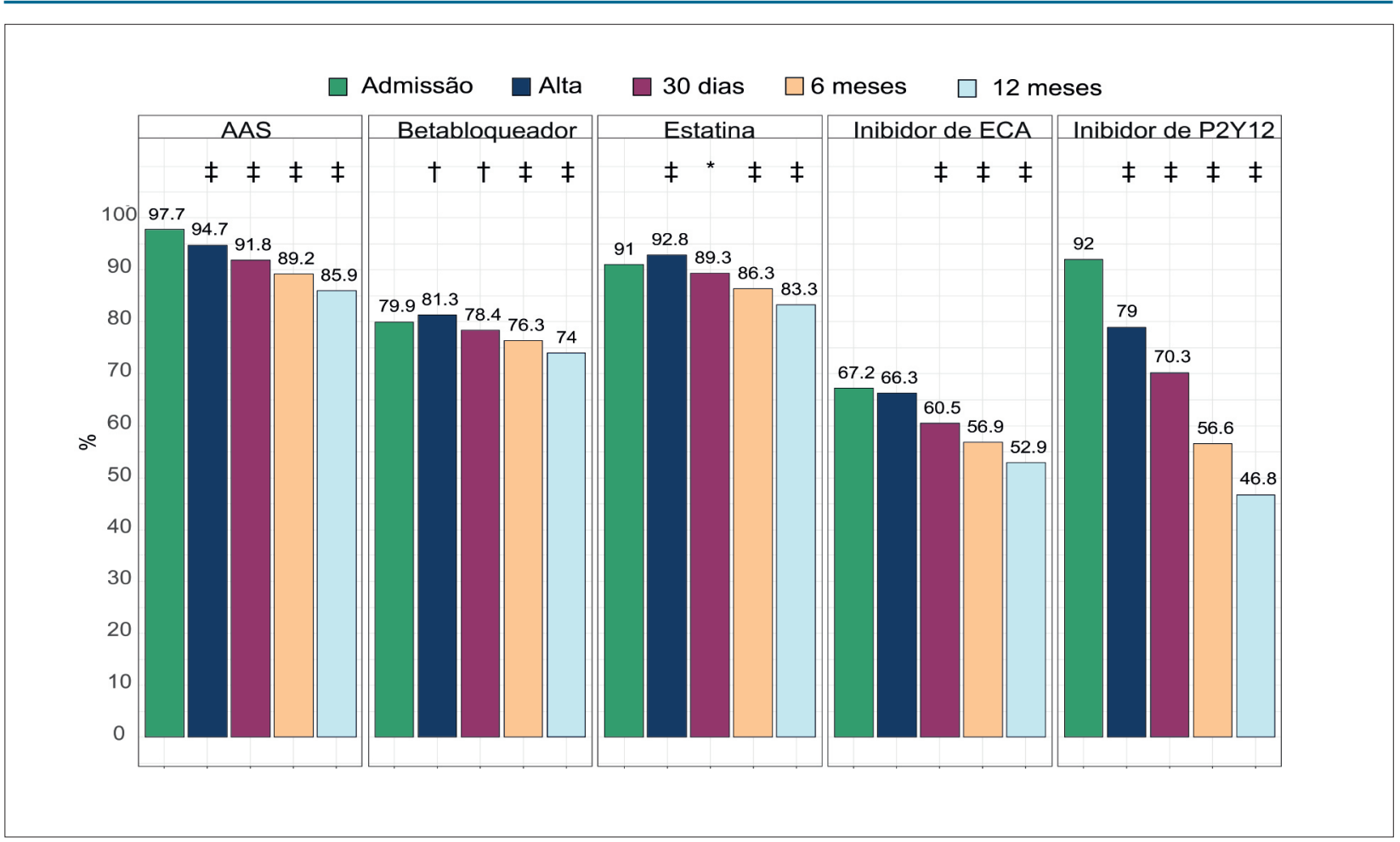

Figura 1 - Adesão a terapias baseadas em evidência no seguimento de 1 ano. Para a comparação da continuidade da prescrição do medicamento nos seguimentos com a admissão, foi ajustado um modelo de Equações de Estimação Generalizada (EEG) para dados binários, para considerar a dependência entre as obsenvações. ¥ Valor de $p<0,001$; Comparação entre o seguimento e a admissão; † Valor de p < 0,01; Comparação entre o seguimento e a admissão; * Valor de $p<0,05$; Comparação entre o seguimento e a admissão.

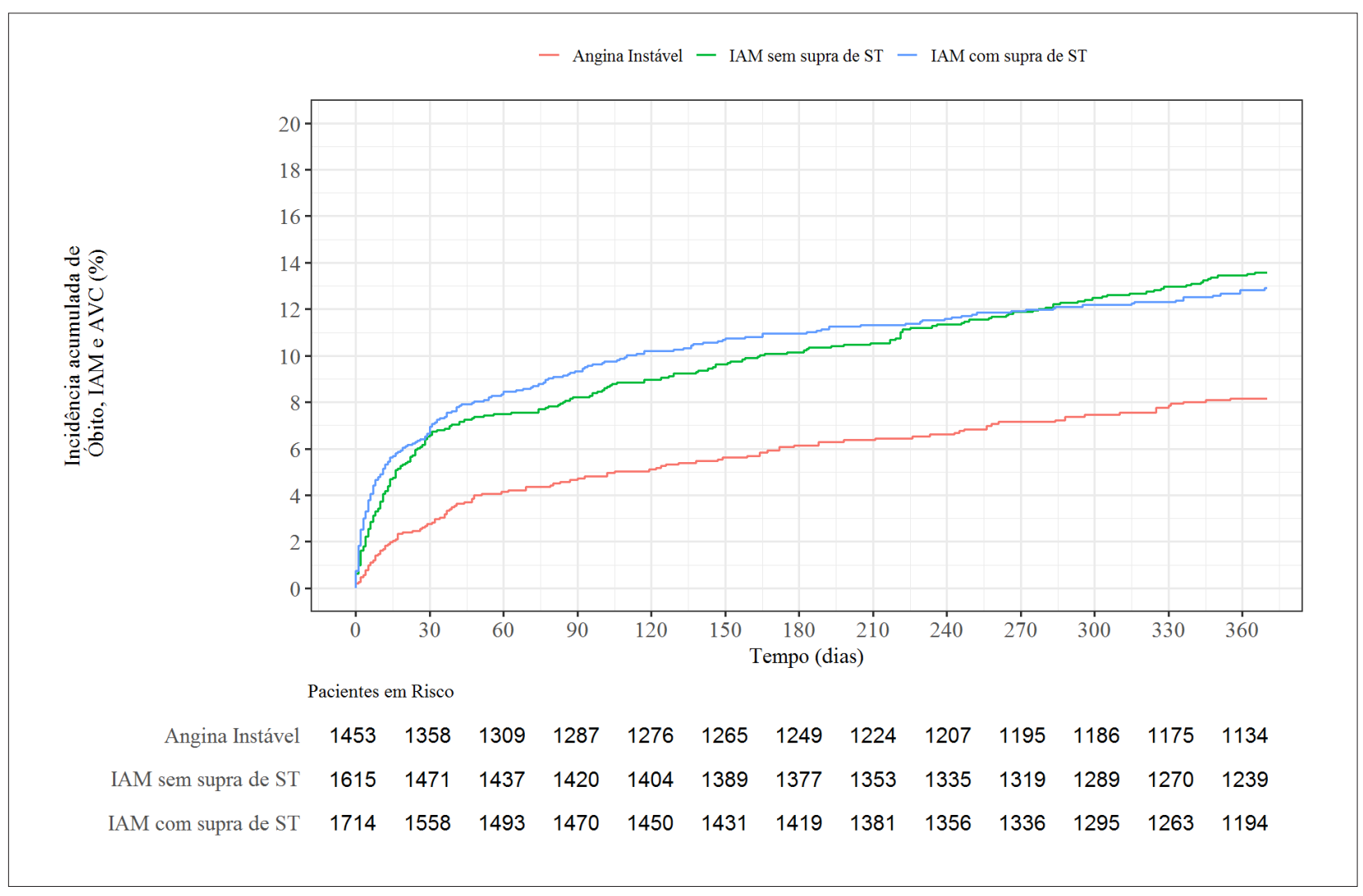

Figura 2-Desfechos clínicos em 1 ano de acordo com o diagnóstico. 
Tabela 3 - Relação entre procedimento de revascularização e desfecho clínico nos 3 tipos de síndrome coronária aguda

\begin{tabular}{|c|c|c|c|}
\hline $\begin{array}{l}\text { Eventos nos pacientes revascularizados em relação aos não- } \\
\text { revascularizados }\end{array}$ & $\begin{array}{l}\text { Angina Instável } \\
\text { HR }[95 \% \text { IC] }\end{array}$ & $\begin{array}{l}\text { IAM sem supra de ST } \\
\qquad \text { HR }[95 \% \text { IC }]\end{array}$ & $\begin{array}{l}\text { IAM com supra de ST } \\
\text { HR }[95 \% \text { IC }]\end{array}$ \\
\hline Sangramento Grave & $2,03[0,75 ; 5,44]$ & $1,15[0,55 ; 2,41]$ & $1,28[0,37 ; 4,50]$ \\
\hline Parada Cardiorrespiratória & $0,27[0,09 ; 0,79]$ & $0,54[0,34 ; 0,87]$ & $0,54[0,36 ; 0,83]$ \\
\hline Reinfarto do miocárdio & $1,69[1,03 ; 2,76]$ & $1,28[0,85 ; 1,90]$ & $0,87[0,53 ; 1,43]$ \\
\hline Acidente vascular encefálico (AVE) & $1,18[0,26 ; 5,28]$ & $0,80[0,30 ; 2,13]$ & $1,02[0,34 ; 3,11]$ \\
\hline Óbito & $0,33[0,17 ; 0,65]$ & $0,53[0,37 ; 0,76]$ & $0,45[0,33 ; 0,63]$ \\
\hline Óbito Cardiovascular & $0,45[0,20 ; 1,06]$ & $0,43[0,28 ; 0,66]$ & $0,43[0,31 ; 0,62]$ \\
\hline Desfecho Combinado & $0,97[0,66 ; 1,42]$ & $0,75[0,57 ; 0,98]$ & $0,64[0,48 ; 0,85]$ \\
\hline
\end{tabular}

Desfecho Combinado: Óbito, Reinfarto do miocárdio e AVE. HR: Hazard Ratio

A taxa de eventos nos usuários do SUS foi de 16,6 por 100 pacientes-ano, ao passo que, na rede privada/convênio, foi de 9,10 por 100 pacientes-ano $(p<0,01)$. Na análise por região, a taxa de óbito em 1 ano foi significativamente maior na região norte $(19,8 \%$; IC95\% 12,6-27,0), seguida pelas regiões sudeste $(8,0 \%$; IC 95\% 7,0-9,1), sul $(6,8 \%$; IC $95 \% 4,8-8,7)$ e nordeste $(5,6 \%$; IC 95\% 3,7-7,5). A região centro-oeste teve menor representação de pacientes com taxa de mortalidade intermediária entre o norte e o restante do país (14,2\%; IC95\% 2,8-25,5). Na comparação dos preditores de eventos entre a região norte e as 3 regiões com taxa de eventos menores (sul, sudeste e nordeste), houve maior frequência de IAM cSST (51,0\% x 35,3\%; p $<0,01)$, atendimento pelo SUS $(100 \% \times 51,8 \%$; p < 0,01) e terapia incompleta nos pacientes incluídos na região norte do país $(47,9 \% \times 37,2 \% ; p<0,01)$.

\section{Discussão}

Neste, que é o maior registro prospectivo brasileiro de pacientes com SCA, observou-se que mais de dois terços dos eventos são classificados como infarto agudo do miocárdio na admissão. O perfil dos pacientes indica predomínio do sexo masculino (70\%), quase um terço de diabéticos e a hipertensão arterial sistêmica como o fator de risco mais comum (74,6\%). Quase $40 \%$ dos pacientes não recebeu pelo menos uma das terapias baseadas em evidência na internação e a conformidade às recomendações variou de acordo com a região, com o tipo de SCA e com a estratégia de revascularização. O risco de eventos cardiovasculares maiores em 1 ano foi de 13,6 por 100 pessoas ano e dos sete fatores associados a estes eventos, dois são relacionados a aspectos do atendimento: financiamento (público x privado) e qualidade da terapia (completa ou não).

A publicação com resultados parciais do ACCEPT feita em $2013^{10}$ tinha incluído 2.584 pacientes e analisado 2.485 , após exclusão de casos não confirmados de SCA. $\mathrm{Na}$ presente análise, foram acrescidos 2.463 pacientes, o que totalizou 5.047 arrolados ao final do estudo (4.782 casos de SCA confirmados). Além do tamanho amostral, uma outra diferença marcante é o tempo de seguimento, pois, assim como a publicação de dados intermediários do
ACCEPT, ${ }^{10}$ a maioria das publicações de registros nacionais em SCA reportaram dados apenas de desfechos intrahospitalares ou de seguimento de 30 dias. ${ }^{12,13}$ O estudo ERICO, publicado em 2015, reportou o seguimento de 1 ano dos pacientes internados por SCA em um hospital público de São Paulo/SP. ${ }^{14}$ Dessa forma, a presente análise incluiu, de forma inédita, dados do seguimento de 12 meses de uma grande população contemporânea de pacientes com SCA de diversas regiões do Brasil, incluindo a análise da conformidade da prescrição médica às recomendações de terapias baseadas em evidência em 12 meses. A adesão inicial da prescrição médica identificada no ACCEPT foi semelhante ao que foi encontrado em registros de países em desenvolvimento, ${ }^{15}$ embora tenha se apresentado abaixo do encontrado em centros que participam de programa de qualidade nos mesmos países. ${ }^{9}$ No seguimento de 1 ano, houve queda na prescrição de todas as terapias, especialmente inibidores de P2Y12, cuja utilização estava bem abaixo daquela identificada em registros internacionais de países desenvolvidos. ${ }^{16,17}$

No seguimento de 12 meses, identificou-se também um risco residual de 13,6 por 100 pacientes-ano para eventos cardiovasculares maiores (reinfarto, morte, AVE). A relação destes eventos com a realização de revascularização pareceu mais clara nos casos de IAM, pois na angina instável a análise combinada dos desfechos CV não mostrou menor taxa nos pacientes submetidos a revascularização. Por ser uma análise observacional (não randomizada), tal evidência não permite estabelecer relação de causa-efeito, mas reforça a validade externa do conceito gerado por ensaios clínicos sobre o benefício de revascularização em pacientes com SCA, especialmente aqueles de maior risco. ${ }^{18,19}$

Uma forma de minimizar o viés de uma análise observacional é incluir as diversas variáveis coletadas em um modelo que permita identificar a relação individual de forma independente. Dentre os fatores identificados em análise multivariada, dois foram relacionados ao atendimento: público x privado e qualidade da terapia (completa ou não). A qualidade da terapia se baseou nas recomendações baseadas em evidência para essa população..$^{6,7}$ A relação de desfecho com qualidade da terapia já foi demonstrada em diversas publicações prévias ${ }^{8,15}$ e apresenta importância adicional para a validade externa 
Tabela 4 - Análise multivariada de fatores associados à ocorrência de eventos combinados (AVE, reinfarto ou óbito)

\begin{tabular}{|c|c|c|}
\hline \multirow[b]{2}{*}{ Variáveis } & \multicolumn{2}{|c|}{ Multivariada } \\
\hline & HR $[95 \%$ IC $]$ & Valor de $\mathrm{p}$ \\
\hline \multicolumn{3}{|l|}{ Idade } \\
\hline Idade (aumento de 5 anos) & $1,16[1,11 ; 1,20]$ & $<0,001$ \\
\hline \multicolumn{3}{|l|}{ Sexo } \\
\hline Feminino & $1,10[0,91 ; 1,33]$ & 0,328 \\
\hline \multicolumn{3}{|l|}{$\begin{array}{l}\text { Atendimento (Saúde Suplementar/ } \\
\text { Particular) }\end{array}$} \\
\hline Saúde Suplementar/Particular & $0,57[0,47 ; 0,69]$ & $<0,001$ \\
\hline \multicolumn{3}{|l|}{ Dislipidemia } \\
\hline Sim & $0,98[0,81 ; 1,19]$ & 0,826 \\
\hline \multicolumn{3}{|l|}{ IAM } \\
\hline Sim & $1,29[1,03 ; 1,63]$ & 0,030 \\
\hline \multicolumn{3}{|l|}{ Angina } \\
\hline Sim & $0,95[0,78 ; 1,16]$ & 0,613 \\
\hline \multicolumn{3}{|l|}{ Hipertensão } \\
\hline Sim & $1,08[0,85 ; 1,36]$ & 0,534 \\
\hline \multicolumn{3}{|l|}{ AVE } \\
\hline Sim & $1,38[1,06 ; 1,80]$ & 0,017 \\
\hline \multicolumn{3}{|l|}{ Insuficiência renal } \\
\hline $\operatorname{Sim}$ & $2,08[1,59 ; 2,71]$ & $<0,001$ \\
\hline \multicolumn{3}{|l|}{ Diabetes } \\
\hline Sim & $1,48[1,23 ; 1,78]$ & $<0,001$ \\
\hline \multicolumn{3}{|l|}{ ICC } \\
\hline Sim & $1,10[0,83 ; 1,45]$ & 0,502 \\
\hline \multicolumn{3}{|l|}{ Intervenção coronária percutânea } \\
\hline Sim & $1,00[0,80 ; 1,27]$ & 0,961 \\
\hline \multicolumn{3}{|l|}{ Cirurgia de RM } \\
\hline Sim & $0,94[0,72 ; 1,25]$ & 0,684 \\
\hline \multicolumn{3}{|l|}{ Uso de AAS } \\
\hline Sim & $1,18[0,96 ; 1,47]$ & 0,120 \\
\hline \multicolumn{3}{|l|}{ Tabagismo } \\
\hline Nunca & ref & ref \\
\hline Ex-tabagista & $1,22[0,99 ; 1,50]$ & 0,055 \\
\hline Atual & $1,27[1,00 ; 1,62]$ & 0,047 \\
\hline \multicolumn{3}{|l|}{ Terapia completa } \\
\hline Sim & $0,72[0,61 ; 0,86]$ & $<0,001$ \\
\hline \multicolumn{3}{|l|}{ Diagnóstico Final } \\
\hline Angina Instável & ref & ref \\
\hline IAM sem supra de ST & $1,76[1,39 ; 2,23]$ & $<0,001$ \\
\hline IAM com supra de ST & $2,04[1,59 ; 2,62]$ & $<0,001$ \\
\hline
\end{tabular}

*Variáveis com valor de $p<0,15$ em análise "univariada" foram candidatas para a análise multivariada, ${ }^{*}$ As variáveis que apresentaram valor de $p>$ 0,15 na análise univariada foram: Transferência de outro serviço, História familiar de doença coronariana, Obesidade Abdominal, Sedentarismo e Doença arterial periférica. dos efeitos identificados em ensaios clínicos controlados. A explicação para a diferença de desfechos identificada entre os pacientes do atendimento público ou privado, poderia ser resultante da diferença na qualidade assistencial. Entretanto, como o modelo multivariado identificou que o atendimento privado apresenta associação com melhores desfechos de forma independente da qualidade da terapia, uma possível explicação seria a própria classe social/educacional dos pacientes. Esta informação não foi coletada para inclusão direta no modelo multivariado da presente análise, mas, em estudos prévios, elas foram identificadas como fatores associados a desfechos clínicos nesta população. ${ }^{15,20}$

\section{Limitações do estudo}

Uma limitação do estudo seria referente ao perfil de pacientes, pois trata-se de um registro voluntário, cujos serviços participantes apresentaram capacidade de pesquisa clínica e, por consequência, os resultados podem não ser aplicáveis a populações que não se enquadrem nestas características (ex: hospitais com estrutura mais limitada). De qualquer maneira, mesmo em centros de maior potencial de qualidade assistencial, foram identificadas lacunas relevantes na aplicação da evidência científica. Outra limitação seria a avaliação da adesão às terapias baseadas em evidência, pois essa análise se baseou na adesão do médico em termos de prescrição de terapias baseadas em evidência. Não foram coletados dados sobre a elegibilidade, a real utilização das terapias prescritas e os motivos para a descontinuação da prescrição. Dessa maneira, tendo em vista que a não adesão por parte dos pacientes não foi avaliada no presente registro, a lacuna sobre o uso de terapias baseadas em evidência poderia ser ainda maior do que aquela encontrada no registro ACCEPT, o qual avaliou a prescrição médica. Finalmente, a análise de desfecho clínico apresenta limitações relacionadas à ausência de adjudicação de eventos e dados faltantes no seguimento de 12 meses de 410 pacientes. Não obstante, a avaliação de desfecho clínico em estudos observacionais pragmáticos habitualmente é realizada por notificação do médico investigador, sem a utilização de um comitê específico para adjudicação, o que representaria um cenário mais próximo da forma de identificação de eventos que ocorre na prática clínica real. Quanto ao seguimento, tendo em vista que as perdas de acompanhamento ocorreram em momentos distintos, as análises foram realizadas por modelo de Cox e, por consequência, os pacientes foram censurados no último contato registrado como forma de minimizar as variações na duração de seguimento.

\section{Conclusão}

No maior registro prospectivo já publicado de pacientes com SCA no Brasil, identificou-se uma taxa média de eventos cardiovasculares maiores em 1 ano acima de 13 por 100 pacientes-ano, mas que atingiu mais de 16,6 por 100 pacientes-ano, no ambiente de atendimento público (SUS). Como há falhas na prescrição de terapias baseadas em evidência desde a admissão, e que se intensificam durante o seguimento, a elaboração de estratégias para aumentar a adesão da prescrição baseada em evidência poderia minimizar o risco de tais eventos na população brasileira. 


\section{Contribuição dos autores}

Concepção e desenho da pesquisa: Barros e Silva PGM, Berwanger O, Guimarães JI, Andrade J, Paola AAV, Malachias MVB, Piva e Mattos LA, Precoma DB, Bacal F, Dutra OP; Obtenção de dados: Barros e Silva PGM, Santos ES, Sousa AC, Cavalcante MA, Andrade PB, Carvalho F, Vargas Filho H; Análise e interpretação dos dados e Redação do manuscrito: Barros e Silva PGM; Obtenção de financiamento: Berwanger O, Guimarães JI, Andrade J, Paola AAV, Malachias MVB, Piva e Mattos LA, Precoma $\mathrm{DB}$, Bacal F, Dutra OP; Revisão crítica do manuscrito quanto ao conteúdo intelectual importante: Berwanger O, Santos ES, Sousa AC, Cavalcante MA, Andrade PB, Carvalho F, Vargas Filho H, Guimarães Jl, Andrade J, Paola AAV, Malachias MVB, Piva e Mattos LA, Precoma DB, Bacal F, Dutra OP.

\section{Aprovação ética e consentimento informado}

Este estudo foi aprovado pelo Comitê de Ética do HCor sob o número de protocolo 117/2010. Todos os procedimentos envolvidos nesse estudo estão de acordo com a Declaração

\section{Referências}

1. World Health Organization (WHO).Cardiovascular Diseases. [Internet]. [Cited in 2019 Dec 12]Available from: http://www.who.int/cardiovascular_diseases/ resources/atlas/en/.

2. Brasil. Ministério da Saúde. [Cited in 2020 Jan 23]. Available from: http://tabnet. datasus.gov.br/cgi/deftohtm.exe?idb2011/c08.def

3. Kochanek KD, XuJQ, MurphySL, Minino AM, Kung HC. Deaths: preliminary data for 2009. Natl Vital Stat Rep.2011;59(4):1-51.

4. Murray CJ, Lopez AD. Alternative projections of mortality and disability by cause 1990-2020: Global Burden of Disease Study. Lancet. 1997;349(9064):1498-504

5. Lloyd-Jones D, Adams R, Carnethon M, De Simone G, Ferguson TB, Flegal K, et al. Heart disease and stroke statistics--2009 update: a report from the American Heart Association Statistics Committee and Stroke Statistics Subcommittee. Circulation. 2009;119(3):480-6.

6. Piegas LS, Feitosa G, Mattos LA, Nicolau JC, Rossi Neto JM, Timerman A, et al. Sociedade Brasileira de Cardiologia. Diretriz da Sociedade Brasileira de Cardiologia sobre Tratamento do Infarto agudo do Miocárdio com Supradesnível do Segmento ST. Arq Bras Cardiol.2009;93(6 supl.2):e179-e264.

7. Nicolau JC, Timerman A, Marin-Neto JA, Piegas LS, Barbosa CJDG, Franci A, Sociedade Brasileira de Cardiologia. Diretrizes da Sociedade Brasileira de Cardiologia sobre Angina Instável e Infarto Agudo do Miocárdio sem Supradesnível do Segmento ST. Arq Bras Cardiol. 2014; 102(3Supl.1):1-61

8. Peterson ED, Roe MT, Mulgund J, deLong ER, Lytle BL, Brindis RG, et al. Association between hospital process performance and outcomes among patients with acute coronary syndromes. JAMA. 2006;295(16):1912-20.

9. de Barros E Silva PGM, Ribeiro HB,Macedo TA, Lopes RD, do Amaral Baruzzi AC, et al. Improvement in quality indicators using NCDR ${ }^{\circledR}$ registries: First international experience. Int J Cardiol. 2018 Sep 15;267:13-5.

10. Piva e Mattos LA, Berwanger O, Santos ES, Reis HJ, Romano ER, Petriz Jl, et al. Clinical outcomes at 30 days in the Brazilian Registry of Acute Coronary Syndromes (ACCEPT). Arq Bras Cardiol. 2013;100(1):6-13.

11. Mattos LA. Rationality and methods of ACCEPT registry - Brazilian registry of clinical practice in acute coronary syndromes of the Brazilian Society of Cardiology. Arq Bras Cardiol. 2011;97(2):94-9. de Helsinki de 1975, atualizada em 2013. O consentimento informado foi obtido de todos os participantes inclú́dos no estudo

\section{Potencial conflito de interesses}

Pedro Gabriel Melo de Barros e Silva declara ter recebido honorários e bolsas de pesquisa da Pfizer, Roche Diagnostics e Bayer. Otavio Berwanger declara ter recebido bolsas de pesquisa e honorários pessoais da ASTRA Zeneca, Bayer e Boehringer Ingelheim; Bolsas da Amgen e Roche Diagnosis; e honorários pessoais da Novo Nordisk e Novartis.

\section{Fontes de financiamento}

O presente estudo foi financiado pela Sociedade Brasieira de Cardiologia.

\section{Vinculação acadêmica}

Não há vinculação deste estudo a programas de pósgraduação.

12. Soeiro AM, Silva PGMBE, Roque EAC, Bossa AS, Biselli B, Leal CAT, et al. Prognostic Differences between Men and Women with Acute Coronary Syndrome. Data from a Brazilian Registry. Arq Bras Cardiol. 2018;111(5):648-53.

13. Nicolau JC, Franken M, Lotufo PA, Carvalho AC, Marin Neto JA, Lima FG, et al. Use of demonstrably effective therapies in the treatment of acute coronary syndromes: comparison between different Brazilian regions. Analysis of the Brazilian Registry on Acute Coronary Syndromes (BRACE). Arq Bras Cardiol. 2012;98(4):282-9.

14. Santos IS, Goulart AC, Brandão RM, Santos RC, Bittencourt MS, Sitnik D, Pereira AC, Pastore CA, Samesima N, Lotufo PA, Bensenor IM. One-year Mortality after an Acute Coronary Event and its Clinical Predictors: The ERICO Study. Arq Bras Cardiol. 2015;105(1):53-64

15. Xavier D, Pais P, Devereaux PJ, Xie C, Prabhakaran D, Reddy KS, et al.Treatment and outcomes of acute coronary syndromes in India (CREATE) a prospective analysis of registry data. Lancet. 2008;371(9622):1435-42.

16. Ferrières J, Sartral M, Tcherny-Lessenot S,Belger M, APTOR trial investigators. A prospective observational study of treatment practice patterns in acute coronary syndrome patients undergoing percutaneous coronary intervention in Europe. Arch Cardiovasc Dis. 2011;104(2):104-14.

17. Bueno H, Pocock S, Danchin N, Annemans L, Grewgson J, Medina J, et al. International patterns of dual antiplatelet therapy duration after acute coronary syndromes. Heart. 2017;103(2):132-8.

18. LiYi, Liu N, Lu J. Outcomes in patients with non-ST-elevation acute coronary syndrome randomly assigned to invasive versus conservative treatment strategies: A meta-analysis. Clinics (Sao Paulo) 2014; 69(6):398-404.

19. Bavry A, Kumbhani DJ, Rassi AN, Bhatt DL, Askari AT. Benefit of early invasive therapy in acute coronary syndromes: a meta-analysis of contemporary randomized clinical trials. J Am Coll Cardiol. 2006;48(7):1319-25.

20. Al-Zakwani I, M Mabry R, Zubaid M, Alsheikh-Alii AA, Almahmeed W Shehab A, et al. Association between education and major adverse cardiac events among patients with acute coronary syndrome in the Arabian Gulf. BMJ Glob Health. 2019;4(1):e001278. 